\title{
Parafunctional habits are associated cumulatively to painful temporomandibular disorders in adolescents
}

\section{Giovana FERNANDES(a) \\ Ana Lúcia FRANCO-MICHELONI(a) José Tadeu Tesseroli de SIQUEIRA ${ }^{(b)}$ Daniela Aparecida Godói GONÇALVES(a) \\ Cinara Maria CAMPARIS(a)}

(a) Universidade Estadual Paulista - UNESP, Araraquara School of Dentistry, Department of Dental Materials and Prosthodontics, Araraquara, SP, Brazil.

(b) Universidade de São Paulo - USP, Hospital das Clínicas - HC, Orofacial Pain Clinic, São Paulo, SP, Brazil.

Declaration of Interests: The authors certify that they have no commercial or associative interest that represents a conflict of interest in connection with the manuscript.

Disclosures: Parts of these data were presented at the 2013 Annual Scientific Meeting of the International Association for Dental Research in Seattle, WA, USA.

\section{Corresponding Author:}

Giovana Fernandes

E-mail: giovana_fernandes@hotmail.com

DOI: 10.1590/1807-3107BOR-2016.vol30.0015

Submitted: Nov 30, 2014

Accepted for publication: Aug 19, 2015

Last revision: Sep 25, 2015
Abstract: This cross-sectional study was designed to evaluate the effect of sleep bruxism, awake bruxism and parafunctional habits, both separately and cumulatively, on the likelihood of adolescents to present painful TMD. The study was conducted on a sample of 1,094 adolescents (aged 12-14). The presence of painful TMD was assessed using the Research Diagnostic Criteria for Temporomandibular Disorders, Axis I. Data on sleep bruxism, awake bruxism and parafunctional habits (nail/pen/pencil/lip/cheek biting, resting one's head on one's hand, and gum chewing) were researched by self-report. After adjusting for potential demographic confounders using logistic regression, each of the predictor variables (sleep bruxism, awake bruxism and parafunctional habits) was significantly associated with painful TMD. In addition, the odds for painful TMD were higher in the concomitant presence of two $(\mathrm{OR}=4.6,[95 \% \mathrm{CI}=2.06,10.37])$ or three predictor $(\mathrm{OR}=13.7,[95 \% \mathrm{CI}=5.72,32.96])$ variables. These findings indicate that the presence of concomitant muscle activities during sleep and awake bruxism and parafunctional habits increases the likelihood almost linearly of adolescents to present painful TMD.

Keywords: Adolescent; Bruxism; Pain; Temporomandibular Joint Disorders.

\section{Introduction}

Painful temporomandibular disorder (TMD) has been viewed as a complex and multidimensional process, involving various psychosocial and environmental factors that may contribute to its pathogenesis. ${ }^{1}$ Adolescence is one of the most dynamic periods of human growth and development, ${ }^{2}$ and seems to relate to an increase in the prevalence for TMD pain, as compared with childhood. ${ }^{3}$ The concern about painful TMD in adolescents is growing, and the prevalence rate has ranged from $0.7 \%$ to $23.5 \%$, up to now. ${ }^{4,5}$

Among the factors involved in painful TMD pathogenesis, the role of sleep bruxism, awake bruxism, and parafunctional habits has been studied extensively in the adult population, ${ }^{6}$ whereas there are few studies on this issue in the pediatric population. ${ }^{7,9}$ In regard to sleep bruxism and awake bruxism, a recent international expert consensus suggested that there must be a clear differentiation between the two. ${ }^{10}$ Sleep bruxism is classified as a sleep-related movement disorder, and defined as an oral activity characterized by grinding or clenching of the teeth during sleep, usually 
associated with sleep arousal. ${ }^{11}$ Awake bruxism is the habit of clenching the teeth during wakefulness, and probably has a different cause and pathophysiology from sleep bruxism. ${ }^{12}$ Despite this conceptual difference, it has been observed that their concomitant presence is frequent and that sleep bruxism significantly increases the odds for awake bruxism and vice-versa. ${ }^{7}$ On the other hand, activities named as parafunctional habits are those that exceed the individual's physiologic tolerance, leading to the breakdown of the masticatory system. ${ }^{13}$ The parafunctional habits commonly reported by adolescents are nail/pen/pencil/and lip/cheek biting, resting their head on their hand, and gum chewing. ${ }^{7}$

It has been shown that sleep and awake bruxism and parafunctional habits are associated with painful TMD. ${ }^{14}$ Moreover, a dose-response gradient has been suggested in this association, i.e., more muscle activity (intensity, duration) leads to more overloading and muscle pain. ${ }^{15}$

Therefore, we think it interesting to study the effect of sleep and awake bruxism and of parafunctional habits, both separately and cumulatively, on the likelihood of adolescents to develop painful TMD.

\section{Methodology}

This was a cross-sectional study conducted on a sample of 12-14-year-old adolescents of a public school from Araraquara, São Paulo, Brazil.

\section{Ethical Aspects}

The study was approved by the Ethics Committee of the Araraquara School of Dentistry, Universidade Estadual Paulista - UNESP (Institutional Ethics Committee approval - protocol number 70/10). All parents or legal guardians gave their written consent to the adolescents to participate in the study.

\section{Sampling Procedures}

The study comprised a total of 7,172 students, aged 12-14 years, registered at Secretaria Estadual da Educação of Araraquara city, and distributed throughout 24 public schools. Based on the total number, the minimal sample size was calculated as 1,005 participants, considering $2 \%$ of TMD pain prevalence in adolescents, taken from a previous international study, ${ }^{16}$ and a sample power of $80 \%$. Considering an absenteeism rate of $20 \%$, the total number of participants to be included in the statistical sample was 1,257.

The 24 public schools were visited mostly between June 2011 and May 2012. Each potential participant received a brief explanation about the aims of the research and a letter to take to their parents or legal guardian that included a brief questionnaire on the participant's socioeconomic status and a consent form. A total of 3,117 adolescents were invited and 1,307 adolescents returned the consent form and participated in the study.

\section{Exclusion criteria}

The exclusion criteria were: 1) individuals with tooth pain; 2) acute facial pain after recent injury;3) individuals receiving orthodontic or TMD treatment; 4) use of medications that affect the central nervous system.

\section{Data Collection}

\section{Outcome variable}

Painful TMD was evaluated by the Axis I physical examination form of the Research Diagnostic Criteria for Temporomandibular Disorders (RDC/TMD), Portuguese version, ${ }^{17}$ together with Axis II questions \#3 ("Have you had pain in your face, jaw, temple, in front of your ear or in your ear in the past month?"), \#4 ("How many years ago did your facial pain begin for the first time?") and \#14 ("Have you ever had your jaw lock or catch, so that it won't open all the way"). The RDC/TMD allows three groups of diagnoses: I - muscle disorders, II - joint disk displacements, and III - other joint conditions. The case group was composed of adolescents with painful TMD diagnoses that included group I and/or III diagnoses: myofascial pain with or without limited opening, TMJ arthralgia and osteoarthritis, either isolated or combined. Adolescents who were not diagnosed with TMD or who were diagnosed with non-painful TMD composed the control group. Non-painful TMD diagnoses included group II and/or III diagnoses: joint disk displacements and TMJ osteoarthrosis, either isolated or combined. Thus, the adolescents diagnosed with painful TMD and those diagnosed with combined painful and non-painful TMD composed the case group. 
Possible demographic confounder variables

Gender and ethnicity: The participant's racial classification was established according to the Brazilian Institute of Geography and Statistics, which names such categories as white, black, mulatto, Asian, and Indian. According to the Instituto Brasileiro de Geografia e Estatística - IBGE, ${ }^{18}$ all Brazilians must declare their own ethnicity. Subsequently, ethnicity was dichotomized into two groups: white and others (black, mulatto, Asian, and Indian).

Menarche: a female investigator inquired into the menarche status of the girls. This factor was evaluated by the question "Have you had your first menstrual period?" The girls could choose between "no" and "yes".

\section{Predictor variables}

Sleep bruxism: the self-report was obtained from the question "Have you been told, or have you noticed that you grind your teeth or clench your jaw while sleeping at night?" (Brazilian-Portuguese RDC/TMD questionnaire). ${ }^{17}$

Awake bruxism: the self-report was obtained from the question "Do you grind your teeth or clench your jaw during the day?" (Brazilian-Portuguese RDC/TMD questionnaire). ${ }^{17}$

Parafunctional habits: the self-report was assessed using the question "Do you have any habits like nail/pen/pencil/lip/cheek biting, resting your head on your hand, and habitual gum chewing?".

\section{Pilot Study and Examiner Training}

A pilot study was conducted prior to the survey, to determine the feasibility and reliability of the data collection procedure. The questionnaire for adolescents and the clinical examination were tested on 77 adolescents (12-14 years old) from one of the eligible schools, on two distinct occasions, with a seven-day interval. The intraexaminer reliability of the RDC/ TMD instrument was determined using Cohen's kappa, yielding values of 0.795 for myofascial pain and 0.884 for arthralgia, scores considered substantial and almost perfect, respectively. Intraclass correlation coefficients (ICC) were calculated to test the questions for the self-reports, using absolute agreement. The ICC's ranged from fair to good to excellent. The reliability for the question on sleep bruxism was considered excellent (ICC $=0.968)$, and that of the questions about awake bruxism and parafunctional habits, fair to good (ICC $=0.69$ and 0.439 , respectively). All the physical examinations performed in both the pilot study and the study itself were done by a single trained examiner, whereas all the questions were applied by another single examiner.

\section{Statistical analysis}

Descriptive statistics and frequency counts were used to characterize the sample. First, the sample was stratified according to painful TMD diagnoses to study the association according to demographic variables (gender and ethnicity), and to identify potential demographic confounders. Second, the association between each possible pair of predictor variables (sleep bruxism, awake bruxism, and parafunctional habits) was evaluated. The Chi-square $\left(\chi^{2}\right)$ odds ratio (OR) with a $95 \%$ confidence interval (CI) was applied to both analyses, and the significance level adopted was 0.05 .

A univariate logistic regression model was used to assess the association between the predictor variables (sleep bruxism, awake bruxism and parafunctional habits) and the outcome variable (painful TMD). The multivariate regression model was adjusted for potential demographic confounders (e.g., gender, menarche and ethnicity) that were analyzed previously.

A second logistic regression model was applied subsequently, using the count of variables as a predictor. In other words, a participant who endorsed none of the predictor variables would have a count of 0 , whereas a participant who endorsed all three had a count of 3 . This count was modeled as a categorical variable. Two logistic regression models were fitted to predict the presence of TMD based on this count variable. The first model was a single logistic regression model that included only the count variable as a predictor variable. The second model was a multiple regression model adjusted for potential demographic confounders.

The data were checked for multicollinearity, using tolerance values $<0.10$ and a variance inflation factor $>10$. All analyses were performed with the SPSS software package, version 21.0 for Mac (SPSS Inc., Chicago, USA). 


\section{Results}

The final sample consisted of 1,307 adolescents who had agreed to participate, had returned the written term of consent, and were present when the evaluations were performed (a response rate of $41.9 \%$ ). According to the exclusion criteria, 213 adolescents were excluded: 178 were undergoing current orthodontic or TMD treatment, and 35 were using medical substances that could act on the central nervous system (e.g. amitriptyline, fluoxetine, Ritalin).

In all, the case group comprised 279 adolescents presenting either painful TMD or a diagnosis (16.8\%) of combined painful and non-painful TMD. The control group was composed of 814 adolescents. Of these, $94.0 \%$ did not present TMD diagnoses, and 6.0\% presented an isolated diagnosis of non-painful TMD.

The total sample consisted of 595 girls (54.4\%) and 499 boys (45.6\%). The majority of the adolescents were Caucasian (67.4\%), and the mean age was 13.7 $( \pm 0.78)$. The prevalence of painful TMD according to gender and ethnicity is depicted in Table 1. Only gender was associated with painful TMD, and was consequently considered a potential demographic confounder.

The association of the predictor variables to one another showed that awake bruxism was strongly associated with parafunctional habits $(\mathrm{OR}=2.8$, $95 \% \mathrm{CI}=1.7,4.8)$. A weaker association was observed between sleep bruxism and awake bruxism $(\mathrm{OR}=1.4$, $95 \% \mathrm{CI}=1.0,1.9)$ and between sleep bruxism and parafunctional habits $(\mathrm{OR}=1.7,95 \% \mathrm{CI}=1.1,2.9)$.
The association between the predictor variables and painful TMD is shown in Table 2. All of them were significantly associated with painful TMD in a multiple logistic regression model. Furthermore, Table 3 shows that the likelihood to develop painful TMD was greater for individuals with a greater number of predictor variables. Individuals who presented two predictor variables, and those who presented all three, had higher odds of having painful TMD [OR $=4.5(95 \% \mathrm{CI}=2.01-10.26) ; \mathrm{OR}=13.3$ $(95 \% \mathrm{CI}=5.67-31.61)]$ than those who presented none or only one predictor variable, after adjusting for gender. There were no signs of multicollinearity.

\section{Discussion}

This study indicates that sleep and awake bruxism, as well as parafunctional habits, are associated with painful TMD, and that concomitant muscle activities from sleep and awake bruxism and parafunctional habits increase the likelihood of adolescents to present painful TMD.

Significant associations between painful TMD and the female sex have also been observed in previous studies involving adolescents..$^{19,20,21}$ Regarding the menarche status, our results are in agreement with a previous Brazilian study reporting that the female gender was significantly associated with TMD, whereas the presence of menarche was not. ${ }^{21}$ Since the age of our sample was limited (12-14 years old), it could be said that we assessed girls at a similar pubertal stage, independently of their menarche

Table 1. Demographic characteristics of the sample and their association with painful TMD.

\begin{tabular}{|c|c|c|c|c|c|c|c|}
\hline & \multicolumn{6}{|c|}{ Group } & \multirow[b]{3}{*}{$95 \% \mathrm{Cl}$} \\
\hline & \multicolumn{2}{|c|}{ Total } & \multirow{2}{*}{$\begin{array}{c}\text { N Control } \\
n(\%)\end{array}$} & \multirow{2}{*}{$\begin{array}{l}\text { Case } \\
\text { n (\%) }\end{array}$} & \multirow[b]{2}{*}{$p$ value } & \multirow[b]{2}{*}{ OR } & \\
\hline & $\mathrm{n}$ & $\%$ & & & & & \\
\hline Gender & & & & & & & \\
\hline Boys & 499 & 45.6 & $386(77.4)$ & $113(22.6)$ & 0.041 & 1.3 & $1.01-1.75$ \\
\hline Girls & 595 & 54.4 & $428(71.9)$ & $167(28.1)$ & & & \\
\hline Menarche & & & & & & & \\
\hline Girls without menarche & 119 & 20.0 & $91(76.5)$ & $28(23.5)$ & 0.218 & 1.3 & $0.84-2.14$ \\
\hline Girls with menarche & 476 & 80.0 & $337(70.8)$ & $139(29.2)$ & & & \\
\hline Race & & & & & & & \\
\hline White & 735 & 67.4 & $547(74.4)$ & $188(25.6)$ & 0.915 & 1.0 & $0.76-1.36$ \\
\hline Others & 355 & 32.5 & $263(74.1)$ & $92(25.9)$ & & & \\
\hline
\end{tabular}


Table 2. Single and multiple logistic regression models for prediction of painful TMD for each predictor variable. All predictor variables are presented as absolute number (n) and percentages (\%) stratified by the presence of painful TMD.

\begin{tabular}{|c|c|c|c|c|c|c|c|c|c|}
\hline \multirow{2}{*}{ Predictor variables } & & \multicolumn{2}{|c|}{ Group } & \multicolumn{3}{|c|}{ Single Regression } & \multicolumn{3}{|c|}{ Multiple Regression* } \\
\hline & & Control n (\%) & Case n (\%) & $p$-value & OR & $95 \% \mathrm{Cl}$ & $p$-value & OR & $95 \% \mathrm{Cl}$ \\
\hline \multirow[t]{2}{*}{ Sleep bruxism } & No & $527(79.7)$ & $134(20.3)$ & \multicolumn{3}{|c|}{ Reference } & \multicolumn{3}{|c|}{ Reference } \\
\hline & Yes & $286(66.2)$ & $146(33.8)$ & 0.000 & 2.0 & $1.53-2.64$ & 0.000 & 2.0 & $1.50-2.65$ \\
\hline \multirow[t]{2}{*}{ Awake bruxism } & No & $591(81.1)$ & $138(18.9)$ & \multicolumn{3}{|c|}{ Reference } & \multicolumn{3}{|c|}{ Reference } \\
\hline & Yes & $222(61.0)$ & $142(39.0)$ & 0.000 & 2.7 & $2.07-3.62$ & 0.000 & 2.6 & $1.93-3.42$ \\
\hline \multirow[t]{2}{*}{ Parafunctional habits } & No & 109 (89.3) & $13(10.7)$ & \multicolumn{3}{|c|}{ Reference } & \multicolumn{3}{|c|}{ Reference } \\
\hline & Yes & $704(72.5)$ & $267(27.5)$ & 0.000 & 3.2 & $1.76-5.75$ & 0.001 & 2.6 & $1.42-4.78$ \\
\hline
\end{tabular}

*Adjusted for gender.

Table 3. Association between the number of predictor variables and painful TMD.

\begin{tabular}{lcccccccc}
\hline \multirow{2}{*}{ Predictor variables } & \multicolumn{2}{c}{ Group } & \multicolumn{3}{c}{ Single Regression } & \multicolumn{2}{c}{ Multiple Regression* } \\
\cline { 2 - 9 } & Control $\mathrm{n}(\%)$ & Case $\mathrm{n}(\%)$ & $\mathrm{p}$-value & OR & $95 \% \mathrm{Cl}$ & $\mathrm{p}$-value & OR & $95 \% \mathrm{Cl}$ \\
\hline 0 & $73(9.0)$ & $7(2.5)$ & & Reference & & Reference \\
1 & $420(51.8)$ & $86(31.3)$ & 0.0871 & 2.1 & $0.95-4.70$ & 0.0752 & 2.1 & $0.95-4.70$ \\
2 & $270(33.3)$ & $119(43.3)$ & 0.0001 & 4.6 & $2.01-10.28$ & 0.0002 & 4.5 & $2.01-10.26$ \\
3 & $48(5.9)$ & $63(22.9)$ & $<0.0001$ & 13.7 & $5.78-32.4$ & $<0.0001$ & 13.3 & $5.67-31.61$ \\
\hline
\end{tabular}

*Adjusted for gender.

status. The presence of reproductive hormones seems to increase the risk of developing pain during the time that girls go through puberty.$^{19}$ However, no evidence has been found up to the present time indicating how sex hormones could affect sensory processing in the trigeminal system, ${ }^{20}$ especially during adolescence.

Considering the association among sleep bruxism, awake bruxism and other parafunctional habits, the results showed a week association between sleep and awake bruxism. In fact, this is supported by the hypothesis that sleep bruxism and awake bruxism seem to be different entities, probably with a different etiology and pathophysiology. ${ }^{10}$ Whereas the role of high levels of stress in sleep bruxism may be poor and controversial, ${ }^{22,23}$ stressful life events seem to exacerbate awake bruxism. ${ }^{23}$ Interestingly, studies on parafunctional habits in adolescents have shown an important association of these habits with stress, ${ }^{23}$ which is considered a risk factor for them. ${ }^{24}$ The strong association between awake bruxism and other parafunctional habits could suggest that they share a similar pathophysiology. However, the present methodology does not allow an in-depth analysis of this topic, and more studies are needed.

The present study confirmed that sleep and awake bruxism, as well as parafunctional habits, are strongly associated with painful TMD. Previous studies with adolescent samples have also reported this association..$^{5,22,23}$ In addition, prospective studies have shown that sleep and awake bruxism could be predictors of signs and symptoms of TMD in adolescents, ${ }^{24}$ increasing with frequency and intensity from adolescence to young adulthood. ${ }^{25}$

Some studies have suggested a dose-response gradient measuring how greater levels of bruxism (intensity, duration) could promote more overloading and pain. ${ }^{15}$ This could be confirmed by the present study, the findings of which showed a cumulative effect of muscle activities on the likelihood of presenting painful TMD. It has been suggested that prolonged and sustained, low intensity chewing, clenching, and grinding movements could promote fatigue and a short-lasting sensation of pain ${ }^{26}$ in health adults.

It may be hypothesized that the pain experienced by patients with parafunctional habits could be analogous to mechanical overload-induced post-exercise muscle 
soreness, ${ }^{27}$ probably caused by the sensitization of muscle nociceptors by endogenous substances. ${ }^{27}$ Possibly, the concomitant presence of sleep bruxism, awake bruxism and parafunctional habits could lead to a greater sensitization of muscle nociceptors. In fact, when this mechanical stimulus is persistent, it may prolong the nociceptive excitability of masticatory muscles, and then sensitize afferent nociceptors. Consequently, this process could contribute to the initiation, maintenance and/or progression of painful TMD.

Nonetheless, some limitations may be identified, and care is needed to properly interpret these results. The present study was designed as a cross-sectional model, and, as such, provides only a possible association and not a cause and effect relationship. In regard to sleep bruxism diagnosis, the self-report has limitations. Polysomnographic records are considered the gold standard, but they are difficult to use for large samples due to high cost and accessibility difficulties. Nevertheless, a recent consensus statement suggested that sleep and awake bruxism self-reports may help in identifying possible bruxism behaviors. ${ }^{10}$ Moreover, sleep and awake self-reports still remain the most suitable approach to gathering data for large samples.,14 However, a large scale polysomnographic study showed that there is an overestimation of the sleep bruxism diagnostic when made with a self-report..$^{28}$ Although the self-report of sleep bruxism, awake bruxism and parafunctional habits has low specificity, the testretest reliability revealed that the questions could be considered reliable for use in this study, even though the question on parafunctional habits may have been hard to interpret, because of the variety of aspects involved. Lastly, a variable as important as sleep quality was not considered in this study. It has been suggested that sleep quality is associated with sleep bruxism and awake bruxism..$^{29}$ Low sleep quality seems to be

\section{References}

1. Maixner W, Diatchenko L, Dubner R, Fillingim RB, Greenspan JD, Knott C, et al. Orofacial Pain Prospective Evaluation and Risk Assessment study--the OPPERA study. J Pain. 2011;12(11 Suppl):T4-11.e1-2. doi:10.1016/j.jpain.2011.08.002

2. Rogol, AD. Roemmich, JN. Clark P. Growth at puberty. J Adolesc Health. 2002;31(6 Suppl):192-200. a potential risk factor for TMD pain. ${ }^{30}$ This variable should be looked into further in future studies.

Our methodology also had important strengths: painful TMD was diagnosed and classified following the RDC/TMD, Axis I gold standard. One trained researcher made all the diagnoses, with kappa values ranging between substantial and almost perfect, thus improving the reliability of the data. In addition, a small age-range sample (12-14 years old), in which all the girls already had menarche was carefully selected in order to homogenize hormonal maturity and body development. ${ }^{2}$ Another consideration is that this study is one of the few that clearly distinguished the forms of bruxism - sleep and awake - and that analyzed them separately, as has been strongly recommended by current literature. ${ }^{10}$ Furthermore, it showed the cumulative effect of sleep and awake bruxism and parafunctional habits on the likelihood of adolescents to present painful TMD ${ }^{31}$.

Finally, it is important to understand the concept of the association presented in this study, in order to avoid overly simplistic strategies when diagnosing and managing TMD, since TMD has been viewed as a complex and multidimensional process in which various psychosocial and environmental factors are of utmost relevance.

\section{Conclusion}

The reported results indicate that the presence of concomitant muscle activities from sleep and awake bruxismand parafunctional habits increases the likelihood of adolescents to present painful TMD almost linearly.

\section{Acknowledgements}

The authors would like to thank the Coordenação de Aperfeiçoamento de Pessoal de Nível Superior - CAPES. In addition, we wish to extend our gratitude to the Araraquara public schools and the adolescent participants for their invaluable contribution to this study.

3. Magnusson T, Egermarki I, Carlsson G. A prospective investigation over two decades on signs and symptoms of temporomandibular disorders and associated variables. A final summary. Acta Odontol Scand. 2005;63(2):99-109.

4. Drangsholt M, LeResche L. Temporomandibular disorder pain. In: Crombie I, Crombie IK, Croft PR, Linton SJ, 
LeResche L, VonKorff M, editors. Epidemiology of pain. Seattle: IASP Press; 1999. p. 203-33.

5. Ebrahimi M, Dashti H, Mehrabkhani M, Arghavani M, Daneshvar-Mozafari A. Temporomandibular disorders and related factors in a group of Iranian adolescents: a crosssectional survey. J Dent Res Dent Clin Dent Prospects. 2011;5(4):123-7.

6. Manfredini D, Lobbezoo F. Relationship between bruxism and temporomandibular disorders: A systematic review of literature from 1998 to 2008. Oral Surg Oral Med Oral Pathol Oral Radiol Endod. 2010;109(6):e26-50. doi:10.1016/j.tripleo.2010.02.013

7. Winocur E, Littner D, Adams I, Gavish A. Oral habits and their association with signs and symptoms of temporomandibular disorders in adolescents: a gender comparison. Oral Surg Oral Med Oral Pathol Oral Radiol Endod. 2006;102(4):482-7.

8. Casanova-Rosado JF, Medina-Solís CE, Vallejos-Sánchez AA, Casanova-Rosado AJ, Hernández-Prado B, Avila-Burgos L. Prevalence and associated factors for temporomandibular disorders in a group of Mexican adolescents and youth adults. Clin Oral Investig. 2006;10(1):42-9.

9. van Selms MKA, Visscher CM, Naeije M, Lobbezoo F. Bruxism and associated factors among Dutch adolescents. Community Dent Oral Epidemiol. 2013;41(4):353-63. doi:10.1111/cdoe.12017

10. Lobbezoo F, Ahlberg J, Glaros AG, Kato T, Koyano K, Lavigne GJ, et al. Bruxism defined and graded: an international consensus. J Oral Rehabil. 2013;40(1):2-4. doi:10.1111/joor.12011

11. American Academy of Sleep Medicine. International Classification of Sleep Disorders. 3rd ed. American Academy of Sleep Medicine, editor. Darien: American Academy of Sleep Medicine; 2014.

12. Winocur E, Uziel N, Lisha T, Goldsmith C, Eli I. Self-reported bruxism - associations with perceived stress, motivation for control, dental anxiety and gagging. J Oral Rehabil. 2011;38(1):3-11. doi:10.1111/j.1365-2842.2010.02118.x

13. Okeson JP. Bell's orofacial pain. 6th ed. Chicago: Quintessence; 2005.

14. Fernandes G, van Selms MKA, Gonçalves DAG, Lobbezoo F, Camparis CM. Factors associated with temporomandibular disorders pain in adolescents. J Oral Rehabil. 2015;42(2):113-9. doi:10.1111/joor.12238

15. Svensson P, Jadidi F, Arima T, Baad-Hansen L, Sessle BJ. Relationships between craniofacial pain and bruxism. J Oral Rehabil. 2008;35(7):524-47. doi:10.1111/j.1365-2842.2008.01852.x

16. LeResche L. Epidemiology of temporomandibular disorders: implications for the investigation of etiologic factors. Crit Rev Oral Biol Med. 1997;8(3):291-305.

17. Dworkin SF, LeResche L. Research diagnostic criteria for temporomandibular disorders: review, criteria, examinations and specifications, critique. J Craniomandib Disord. 1992;6(4):301-55.

18. Instituto Brasileiro de Geografia e Estatística. Estudos e Análises: informação demográfica e socioeconômica. 2nd ed. Rio de
Janeiro: IBGE; 2013. Características étnico - raciais da população: classificação e identidades; p. 83-99.

19. LeResche L, Mancl LA, Drangsholt MT, Saunders K, Von Korff M. Relationship of pain and symptoms to pubertal development in adolescents. Pain. 2005;118(1-2):201-9.

20. Cairns BE. The influence of gender and sex steroids on craniofacial nociception Headache. 2007;47(2):319-24.

21. Pereira L, Pereira-Cenci T, Del Bel Cury AA, Pereira S, Pereira A, Ambosano G, et al. Risk indicators of temporomandibular disorder incidences in early adolescence. Pediatr Dent. 2010;32(4):324-8.

22. Emodi-Perlman A, Eli I, Friedman-Rubin P, Goldsmith C, Reiter S, Winocur E. Bruxism, oral parafunctions, anamnestic and clinical findings of temporomandibular disorders in children. J Oral Rehabil. 2012;39(2):126-35. doi:10.1111/j.1365-2842.2011.02254.x

23. Carra MC, Huynh N, Morton P, Rompré PH, Papadakis A, Remise C, et al. Prevalence and risk factors of sleep bruxism and wake-time tooth clenching in a 7- to 17-yr-old population. Eur J Oral Sci. 2011;119(5):386-94. doi:10.1111/j.1600-0722.2011.00846.x

24. Carlsson GE, Egermark I, Magnusson T. Predictors of bruxism, other oral parafunctions, and tooth wear over a 20-year follow-up period. J Orofac Pain. 2003;17(1):50-7.

25. Serra-Negra JM, Tirsa-Costa D, Guimarães FH, Paiva SM, Prodeus IA. Evaluation of parents/guardian knowledge about the bruxism of their children: family knowledge of bruxism. J Indian Soc Pedod Prev Dent. 2013;31(3):153-8. doi:10.4103/0970-4388.117965

26. Koutris M, Lobbezoo F, Naeije M, Wang K, Svensson P, Arendt-Nielsen L, et al. Effects of intense chewing exercises on the masticatory sensory-motor system. J Dent Res. 2009;88(7):658-62. doi:10.1177/0022034509338573

27. Mense S. Nociception from skeletal muscle in relation to clinical muscle pain. Pain. 1993;54(3):241-89.

28. Maluly M, Andersen ML, Dal-Fabbro C, Garbuio S, Bittencourt L, Siqueira JTT, et al. Polysomnographic study of the prevalence of sleep bruxism in a population sample. J Dent Res. 2013;92(7 Suppl):97S-103S. doi:10.1177/0022034513484328

29. Serra-Negra JM, Paiva M, Auad S, Ramos-Jorge M, Pordeus I. Sleep bruxism, awake bruxism and sleep quality among Brazilian dental students: a cross sectional study. Braz Dent J. 2014;25(3):241-7

30. Serra-Negra JM, Scarpelli AC, Tirsa-Costa D, Guimarães FH, Pordeus IA, Paiva SM. Sleep bruxism, awake bruxism and sleep quality among Brazilian dental students: a cross sectional study. Braz Dent J. 2014;25(3):241-7. doi:10.1590/0103-6440201302429

31. Drabovicz PVSM, Salles V, Drabovicz PEM, Fontes MJF. Assessment of sleep quality in adolescents with temporomandibular disorders. J Pediatr. 2012;88(2):169-72. doi:10.2223/JPED.2180 